\title{
Synthesis of Approximating State Feedback for Robotic Manipulator
}

\author{
Dimitri Mahayana \\ School of Electrical Engineering and Informatics, Bandung Institute of Technology \\ dmahayana@stei.itb.ac.id
}

\begin{abstract}
This paper invents a methodology to synthesize proportional derivative controller for robotic manipulator. The proposed design methodology is based on exact feedback linearization method. A proportional derivative controller candidate which linearly approximates the nonlinear controller which is resulted from exact feedback linearization method is evaluated under Lyapunov stability condition. We show the effectiveness of the proposed method by applying it to robotic manipulator model, obtaining the proportional derivative controller, simulating the closed loop behavior of the system. A comparison of the proposed method with exact feedback linearization method shows that there is no significant performance degradation when approximating exact feedback linearization by the proposed method.
\end{abstract}

Keywords: Robotic manipulator, exact feedback linearization, approximating state feedback, Lyapunov stability condition

\section{Introduction}

Robots are ideal candidates for material handling operations, manufacturing, and measuring devices because of their capacity to pick up, move, and release an object, to manipulate both objects and tools and their capacity to explore the three dimensional space.

Nowadays, robotic manipulator are extensively used in the industrial field. The desire of a high-speed or a high-precision performance for this kind of mechanical systems has led to research into improved control systems. These high performance control systems need, in general, the dynamical model of the robotic manipulator in order to generate the control input (Yurkovich, 1992).

Robotic manipulators are highly nonlinear, highly time-varying, and highly coupled. Moreover, there always exists uncertainty in the system model such as external disturbances, parameter uncertainty, sensor errors and so on, which cause unstable performance in the robotic system (Sadati et al, 2005). During the past decade, several design methods, e.g., robust control (Torres et al, 2007), optimal control (Shiller and Dubowsky, 1985), adaptive control (Yazarel and Cheah, 2002), backstepping control (Lotfazar et al, 2003), neural network (Patino et al, 2002), fuzzy logic (Kim et al, 2001), and sliding mode control (Purwar, 2007) for robotic manipulator control have been proposed. In addition, a feedback linearization method (Bedrossian, and Spong, 1995) was proposed to control robotic manipulator. By using Riemannian curvature factorization of the robot inertia matrix to generate feedback linearization, a nonlinear control law is derived.

Feedback linearization is a control design approach for nonlinear systems which attracted lots of research in recent years (Fattah, 2000; Mokhtari et al, 2006; Spong and Groeneveld, 1997). The central idea is to algebraically transform nonlinear systems dynamics into (fully or partially) linear ones, so that linear control techniques can be applied. In the standard approach to exact feedback linearization, one uses coordinate transformation and static state feedback such that the closed-loop system, in the defined region, takes a linear canonical form. After the system's linearization form is obtained, the linear control design scheme is employed to achieve stabilization or tracking (Isidori, 1995; Slotine and Li, 1991).

Received: February $24^{\text {th }}, 2011$. Accepted: Septem ber $22^{\text {nd }}, 2011$ 
In the above exact feedback linearization, the controller characteristics have nonlinear functions such as multiplications of the state variables, polynomial functions, trigonometric functions, and so on, which the implementation of the controllers by using electronic devices have many difficulties (Gray and Meyer, 1977; Mahayana, 1991; Nurbambang and Mahayana, 1990; Rangan et al, 1992). On the other hand, many researchers have proven that the performance of the controllers still maintain good response although the approach of the exact feedback linearization have been used (Chong et al, 1991; Ogaw a et al, 1991).

In (Mahayana, 1998) has been developed a synthesis of nonlinear control system to find a control methodology that makes the exact linearization controller more realizable, but without any significant performance degradation. Instead of the exact controller, the proposed controller was a general form of controller candidates which replace the function of the exact controller. The closed loop stability of the nonlinear system under the controller was evaluated by using the Lyapunov stability theory. The condition under which the origin of the closed loop system being asymptotically stable was derived by characteristic value shift theorem.

In the simulation experiments of this paper, we synthesize the approximating state feedback for robotic control system.

2. Dynamics of Robotic Manipulator

Let us assume that the manipulator of Figure 1 is in the horizontal plane $\{\boldsymbol{G}(\boldsymbol{q})=\mathbf{0}\}$ (Slotine and Li, 1991). The dynamics model of two-link robotic manipulator can be written explicitly as

$$
H(q) \ddot{q}+C(q, \dot{q}) \dot{q}=\tau
$$

where $\boldsymbol{q}=\left[\begin{array}{ll}q_{1} & q_{2}\end{array}\right]^{T}$ is the joint position vector; $\boldsymbol{H}(\boldsymbol{q}) \in \mathfrak{R}^{n \times n}$ denotes the moment of inertia; $\boldsymbol{C}(\boldsymbol{q}, \dot{\boldsymbol{q}}) \dot{\boldsymbol{q}}$ are the Coriolis and centripetal forces; $\boldsymbol{\tau}=\left[\begin{array}{ll}\tau_{1} & \tau_{2}\end{array}\right]^{T}$ is the applied torque vector; $\boldsymbol{H}$ and $\boldsymbol{C}$ in (1) can be described as

$$
\begin{aligned}
& \boldsymbol{H}(\boldsymbol{q})=\left[\begin{array}{ll}
H_{11} & H_{12} \\
H_{21} & H_{22}
\end{array}\right], \\
& \boldsymbol{C}(\boldsymbol{q}, \dot{\boldsymbol{q}})=\left[\begin{array}{cc}
-h \dot{q}_{2} & -h\left(\dot{q}_{1}+\dot{q}_{2}\right) \\
h \dot{q}_{1} & 0
\end{array}\right],
\end{aligned}
$$

where

$$
\begin{aligned}
& H_{11}=a_{1}+2 a_{3} \cos q_{2}+2 a_{4} \sin q_{2} \\
& H_{12}=H_{21}=a_{2}+a_{3} \cos q_{2}+a_{4} \sin q_{2} \\
& H_{22}=a_{2} \\
& h=a_{3} \sin q_{2}-a_{4} \cos q_{2}
\end{aligned}
$$

with

$$
\begin{aligned}
& a_{1}=I_{1}+m_{1} l_{\mathrm{c} 1}^{2}+I_{\mathrm{e}}+m_{\mathrm{e}} l_{\mathrm{ce}}^{2}+m_{\mathrm{e}} l_{1}^{2} \\
& a_{2}=I_{\mathrm{e}}+m_{\mathrm{e}} l_{\mathrm{ce}}^{2} \\
& a_{3}=m_{\mathrm{e}} l_{1} l_{\mathrm{ce}} \cos \delta_{\mathrm{e}} \\
& a_{4}=m_{\mathrm{e}} l_{1} l_{\mathrm{ce}} \sin \delta_{\mathrm{e}}
\end{aligned}
$$




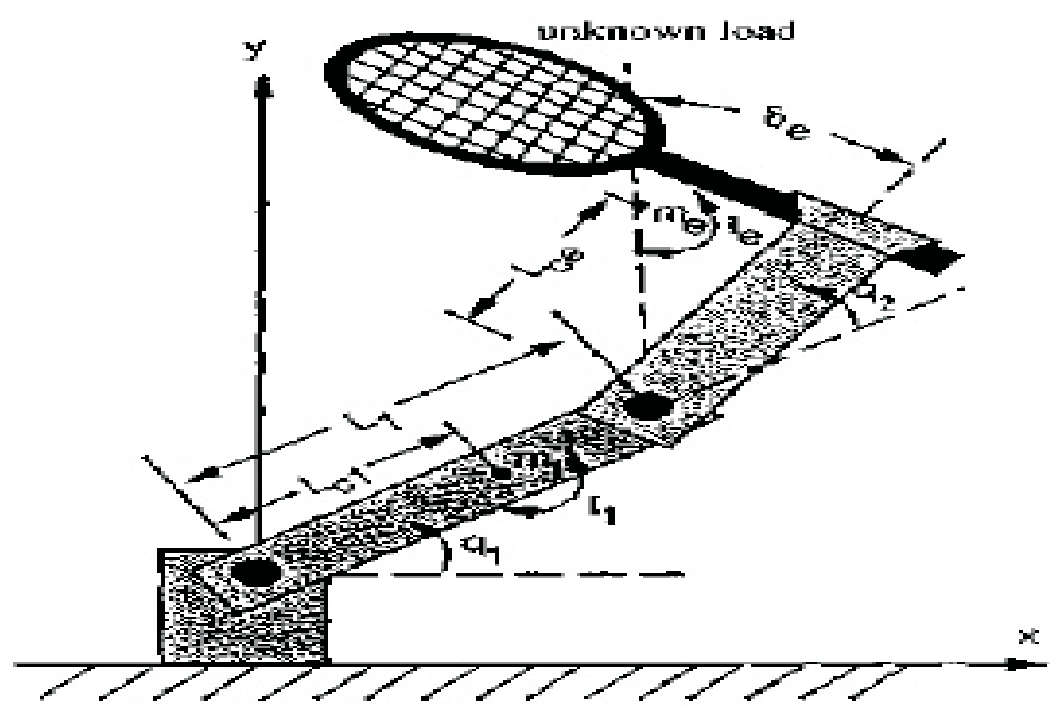

Figure 1

An articulated two-links manipulator

The inertial matrix $\boldsymbol{M ( q )}$ is symmetric and positive definite. It is also bounded as a function of $\boldsymbol{q}: \mu_{1} \boldsymbol{I} \leq \boldsymbol{M}(\boldsymbol{q}) \leq \mu_{2} \boldsymbol{I} . \dot{\boldsymbol{M}}(\boldsymbol{q})-\mathbf{2} \boldsymbol{B}(\boldsymbol{q}, \dot{\boldsymbol{q}})$ is skew symmetric matrix, that is, $\boldsymbol{x}^{T}[\dot{\boldsymbol{M}}(\boldsymbol{q})-\mathbf{2} \boldsymbol{B}(\boldsymbol{q}, \dot{\boldsymbol{q}})] \boldsymbol{x}=0$, where $\boldsymbol{x} \in \mathfrak{R}^{n \times 1}$ is a nonzero vector.

Define $\boldsymbol{u}=\boldsymbol{\tau}$ and state variable $\boldsymbol{x}=\left[\begin{array}{llll}x_{1} & x_{2} & x_{3} & x_{4}\end{array}\right]^{T}=\left[\begin{array}{llll}\theta_{1} & \dot{\theta}_{1} & \theta_{2} & \dot{\theta}_{2}\end{array}\right]^{T}$, the equations of motion for the robotic manipulator can be put in the form of following nonlinear state space:

$$
\dot{x}=f(x)+g(x) u
$$

where $\boldsymbol{f}(\boldsymbol{x})$ is a nonlinear continuous function whose upper bound is known as $|\boldsymbol{f}(\boldsymbol{x})| \leq \boldsymbol{f}_{\max }, \boldsymbol{g}(\boldsymbol{x})$ is a gain function with lower bound $\boldsymbol{g}_{\min }, 0<\boldsymbol{g}_{\min } \leq \boldsymbol{g}(\boldsymbol{x})$.

\section{Controller Synthesis}

\section{A. Matrix Norm and Spectral Radius}

Definition 1. (Goldberg, 1992; Lancaster and Tismenetsky, 1961) If $\boldsymbol{A} \in \boldsymbol{C}^{n \times n}$, then spectral norm of $\boldsymbol{A}$ will be defined as

$$
\|A\|_{\mathrm{s}} \stackrel{\text { def }}{=} \sup _{\boldsymbol{w} \in \boldsymbol{C}^{\mathrm{n}}} \frac{\|\boldsymbol{A} \boldsymbol{w}\|_{2}}{\|\boldsymbol{w}\|_{2}}
$$

Definition 2. (Goldberg, 1992; Lancaster and Tismenetsky, 1961) Spectral radius of a square matrix $\boldsymbol{A} \in \boldsymbol{C}^{n \times n}, \rho(\boldsymbol{A})$, is the maximum among the absolute values of the characteristic values of the matrix $\boldsymbol{A}$.

To compute spectral norm value of a square matrix, we depart from (Lancaster and Tismenetsky, 1961) 


$$
\|\boldsymbol{A}\|_{\mathrm{s}}=\left(\rho\left(\boldsymbol{A} \boldsymbol{A}^{*}\right)\right)^{1}
$$

where subscript $*$ denotes conjugate transpose of a matrix.

\section{B. Lyapunov Theory and Linearization \\ Consider nonlinear system of the form $\dot{\boldsymbol{x}}=\boldsymbol{f}(\boldsymbol{x})$,}

with $\boldsymbol{f}(\mathbf{0})=\mathbf{0}$, or in other words the origin of system is the equilibrium point, and $\boldsymbol{f}$ is a continuous vector field and at least once differentiable with respect to $\boldsymbol{x}$, then the system can be approximated by using a linear time invariant system as follows,

$$
\dot{\boldsymbol{x}}=\boldsymbol{\Psi} \boldsymbol{x}
$$

$$
\boldsymbol{\Psi}=\left.\frac{\partial \boldsymbol{f}}{\partial \boldsymbol{x}}\right|_{\boldsymbol{x}=\mathbf{0}}
$$

where $\boldsymbol{\Psi} \in \mathfrak{R}^{n \times n}$ is a constant matrix.

Theorem 1. (Khalil, 1992; La Salle and Lefschetz, 1961) If the origin $(\boldsymbol{x}=\mathbf{0})$ of the linearization result system (7) is asymp totically stable, then the origin of the original system (6) will be asymptotically stable.

\section{The method of shifting characteristic values}

In this sub-section will be derived the sufficient condition for controller to make the system be asymptotically stable, if the closed loop system under exact controller has an asymptotically stable origin. Let the controller candidate can be expressed as

$$
\boldsymbol{u}_{a} \stackrel{\operatorname{def}}{=} \boldsymbol{u}_{a}(\boldsymbol{x})
$$

and $\boldsymbol{u}_{a}(\boldsymbol{x})$ at least once differentiable with respect to $\boldsymbol{x}$ and $\boldsymbol{u}_{a}(\mathbf{0})=u(\mathbf{0})$.

The error between exact controller and controller candidate is

$$
\boldsymbol{e}(\boldsymbol{x})=\boldsymbol{u}_{a}(\boldsymbol{x})-\boldsymbol{u}(\boldsymbol{x})
$$

Assume a notation

$$
\boldsymbol{A}_{c}=\boldsymbol{A}+\boldsymbol{B} \boldsymbol{K}
$$

with $\boldsymbol{A}_{\boldsymbol{c}}$ is closed loop system matrix with exact controller, and also be defined respectively, several variables as follows: 


$$
\kappa=\left\|\left(\left.\left(\frac{\partial \varepsilon(\boldsymbol{x})}{\partial \boldsymbol{x}}\right)^{T}(\nabla \boldsymbol{T}(\boldsymbol{x}))^{-1}\right|_{\boldsymbol{x}=\mathbf{0}}\right)^{T}\right\|
$$

with $\boldsymbol{P}$ is transformation matrix which transforms $\boldsymbol{A}_{\boldsymbol{c}}$ to its diagonal canonical form;

$$
\boldsymbol{P} \boldsymbol{A}_{c} \boldsymbol{P}^{-1}=\operatorname{diag}\left(\lambda_{1}, \lambda_{1}, \cdots, \lambda_{n}\right)
$$

with $\lambda_{1}, \lambda_{1}, \cdots, \lambda_{n}$ are characteristic values of closed loop matrix with exact controller $\boldsymbol{A}_{\boldsymbol{c}}$, the shortest distance to imaginary axis is noted by $\lambda_{c}$ (by assumption that characteristic values lie in the strict left half of the complex plane).

Since the linear system from exact feedback linearization in the Brunovsky canonical form, then the closed loop system can be made such that all its characteristic values are different. This can be done, e.g., by using pole placement method (Chen, 1970). The assumption of all different characteristic values of $\boldsymbol{A}_{\boldsymbol{c}}$ is necessary to make the transformation of matrix $\boldsymbol{A}_{\boldsymbol{c}}$ to diagonal canonical form can be done (Boothby, 1975; Lancaster and Tismenetsky, 1961).

Theorem 2. If

$$
\operatorname{Re}\left(\lambda_{c}\right)+\kappa v\left(\boldsymbol{A}_{c}\right)<0
$$

then the origin of the system (3) under control input $\boldsymbol{u}_{a}(\boldsymbol{x})$ will be asymptotically stable.

Proof. Theorem 2 will be proved in many stages: construction under exact controller, existence of controller candidate, transformation of system under controller candidate to new state space coordinate, Lyapunov stability analysis, and analysis of shifting characteristic value.

\section{Construction under exact controller}

Equation (1) can be linearized by choosing $\boldsymbol{\tau}$ appropriately. Taking $\boldsymbol{\tau}$ of the form (Slotine and $\mathrm{Li}, 1991)$

$$
\tau=H(q) v+C(q, \dot{q}) \dot{q}
$$

where $\boldsymbol{v} \in \mathfrak{R}^{2 \times 1}$ is the new control input, leads to

$$
\ddot{q}=v
$$

By choosing state variable $\boldsymbol{x}=\left[\begin{array}{llll}x_{1} & x_{2} & x_{3} & x_{4}\end{array}\right]^{T}=\left[\begin{array}{llll}\theta_{1} & \dot{\theta}_{1} & \theta_{2} & \dot{\theta}_{2}\end{array}\right]^{T}$, it can be seen that the robotic manipulator dynamics can be expressed in the Brunovsky canonical form as

$$
\dot{\boldsymbol{x}}=\left[\begin{array}{llll}
0 & 1 & 0 & 0 \\
0 & 0 & 0 & 0 \\
0 & 0 & 0 & 1 \\
0 & 0 & 0 & 0
\end{array}\right] \boldsymbol{x}+\left[\begin{array}{ll}
0 & 0 \\
1 & 0 \\
0 & 0 \\
0 & 1
\end{array}\right] \boldsymbol{v}
$$


Define $z_{i}^{a}=\left[\theta_{\mathrm{i}} \dot{\theta}_{\mathrm{i}}\right]^{T}, \mathrm{i}=1,2$; equation (19) can be put in the form of following three linear subsystems:

$$
\dot{\boldsymbol{z}}_{i}^{a}=\left(\begin{array}{ll}
0 & 1 \\
0 & 0
\end{array}\right) \boldsymbol{z}_{i}^{a}+\left(\begin{array}{l}
0 \\
1
\end{array}\right) v_{\mathrm{i}}
$$

\section{Letting}

$$
v_{\mathrm{i}}=-\lambda_{1, \mathrm{i}} \varsigma_{\mathrm{i}}-\lambda_{2, \mathrm{i}} \dot{\varsigma}_{\mathrm{i}}
$$

The parameters of the new control input, $v_{1}$ are chosen such that $\lambda_{1,1}=90$ and $\lambda_{2,1}=19$ (which correspond to closed-loop poles of the joint angle 1 of -9, - 10), and the parameters of the new control input, $v_{2}$ are chosen such that $\lambda_{1,2}=132$ and $\lambda_{2,2}=23$ (which correspond to closed-loop poles of the joint angle 2 of - 11, - 12).

The feedback gain $\boldsymbol{K}$ can be computed as follows:

$$
\boldsymbol{K}^{T}=\left[\begin{array}{cc}
-90 & 0 \\
-19 & 0 \\
0 & -132 \\
0 & -23
\end{array}\right]
$$

\section{E. Existence of controller candidate}

Since $\boldsymbol{T}: \Omega \rightarrow \boldsymbol{T}(\Omega) \subset \mathfrak{R}^{n}$ with an open set $\Omega$ on $\mathfrak{R}^{n}$ is a diffeomorphism, then $\boldsymbol{T}$ is smooth. Furthermore $\boldsymbol{u}(\boldsymbol{x})$ will be smooth. Based on the smoothness of $\boldsymbol{u}(\boldsymbol{x})$, we can choose a new control input $\boldsymbol{u}_{a}(\boldsymbol{x})$ that is continuous and at least once differentiable with respect to $\boldsymbol{x}$ with $\boldsymbol{u}_{a}(\mathbf{0})=\boldsymbol{u}(\mathbf{0})$, and it satisfies

$$
\left\|u_{a}(\boldsymbol{x})-\boldsymbol{u}(\boldsymbol{x})\right\|_{\infty} \leq \delta
$$

with $\delta$ is a positive constant, at a range $v \subset \Omega \subset \mathfrak{R}^{n}$ where $v$ is a bounded closed set.

\section{F. System transformation under controller candidate}

Nonlinear dynamical system with control input $\boldsymbol{u}_{a}(\boldsymbol{x})$ is

$$
\dot{x}=f(x)+g(x) u_{a}(x)
$$

Equation (24) can be arranged as

$$
\dot{x}=f(x)+g(x)\{u(x)+e(x)\}
$$

After some complex calculations, Equation (25) can be written in new state variable, $\boldsymbol{z}$, as follows 


$$
\dot{\boldsymbol{z}}=\boldsymbol{A}_{c} \boldsymbol{z}+\left[\begin{array}{c}
0 \\
0 \\
\vdots \\
1
\end{array}\right] \boldsymbol{\varepsilon}\left\{\boldsymbol{T}^{-1}(\boldsymbol{z})\right\}
$$

The dynamic of system under controller candidate in new state space coordinate can be seen as a nonlinear system that consists of a linear part and a nonlinear perturbation.

Since $\boldsymbol{u}_{a}(\boldsymbol{x})$ has been chosen such that $\boldsymbol{u}_{a}(\mathbf{0})=\boldsymbol{u}(\mathbf{0})=\mathbf{0}$, then $\boldsymbol{e}(\mathbf{0})=\mathbf{0}$, furthermore at $\boldsymbol{z}=\mathbf{0}$

$$
\boldsymbol{A}_{c} \boldsymbol{z}+\left[\begin{array}{c}
0 \\
0 \\
\vdots \\
1
\end{array}\right] \boldsymbol{\varepsilon}\left\{\boldsymbol{T}^{-1}(\boldsymbol{z})\right\}=\mathbf{0}
$$

This is shown that the equilibrium point of the system under controller candidate is same with the equilibrium point of the system under the exact controller.

G. Lyapunov Stability Analysis

Nonlinear system in Equation (26) can be expressed as

$$
\dot{\boldsymbol{z}}=\boldsymbol{f}_{c}(\boldsymbol{z})
$$

with

$$
\boldsymbol{f}_{c}(\boldsymbol{z})=\boldsymbol{A}_{c} \boldsymbol{z}+\left[\begin{array}{c}
0 \\
0 \\
\vdots \\
1
\end{array}\right] \boldsymbol{\varepsilon}\left\{\boldsymbol{T}^{-1}(\boldsymbol{z})\right\}
$$

Since $\boldsymbol{f}_{c}(\boldsymbol{z})$ is smooth in a neighborhood of the origin then there exists $\frac{\partial \boldsymbol{f}_{c}(\boldsymbol{z})}{\partial \boldsymbol{z}}$ in a neighborhood of the origin. The origin is an equilibrium point, since based on (27)

$$
\boldsymbol{f}_{c}(\mathbf{0})=\mathbf{0}
$$

Linearization of (28) in a neighborhood of the origin yields

$$
\dot{\boldsymbol{z}}=\left.\frac{\partial \boldsymbol{f}_{c}(\boldsymbol{z})}{\partial \boldsymbol{z}}\right|_{z=0} \cdot \boldsymbol{z}
$$


with

$$
\boldsymbol{D}=\left[\begin{array}{c}
\boldsymbol{O}_{(n-1) \times n} \\
------------ \\
\left.\left(\frac{\partial \varepsilon(\boldsymbol{x})}{\partial \boldsymbol{x}}\right)^{T}(\nabla \boldsymbol{T}(\boldsymbol{x}))^{-1}\right|_{\boldsymbol{x}=\mathbf{0}}
\end{array}\right]
$$

According to the Lyapunov stability theory, if the origin of system (32) is asymptotically stable then the origin of system (28) that is a closed loop system under controller candidate $\boldsymbol{u}_{a}(\boldsymbol{x})$, will be asymptotically stable. This can be achieved if all characteristic values of matrix $\left(\boldsymbol{A}_{c}+\boldsymbol{D}\right)$ lie in the strict left half of the complex plane.

\section{H. Analysis of shifting characteristic values}

The linear system (32) can be described by the sum of a nominal system matrix, $\boldsymbol{A}_{\boldsymbol{c}}$ and a perturbation matrix, $\boldsymbol{D}$. Assume $\zeta_{1}, \zeta_{2}, \cdots, \zeta_{n}$ are characteristic values of $\left(\boldsymbol{A}_{c}+\boldsymbol{D}\right)$, by definition of characteristic value (Goldberg, 1992; Lancaster and Tismenetsky, 1961), it can be written as follows

$$
\left(\boldsymbol{A}_{c}+\boldsymbol{D}\right) y_{i}=\zeta_{i} y_{i}, \exists y_{i} \neq 0, y_{i} \in \boldsymbol{C}^{n}
$$

Assume $\boldsymbol{A}_{\boldsymbol{c}}=\boldsymbol{P} \boldsymbol{A}_{\boldsymbol{c}_{\boldsymbol{D}}} \boldsymbol{P}^{-1}$, with $\boldsymbol{A}_{\boldsymbol{c}_{\boldsymbol{D}}}=\operatorname{diag}\left(\lambda_{1}, \lambda_{2}, \cdots, \lambda_{n}\right)$. Equation (34) can be written as follows

$$
\left(\boldsymbol{A}_{\boldsymbol{c}_{\boldsymbol{D}}}+\boldsymbol{P}^{-1} \boldsymbol{D P}\right)_{i}=\zeta_{i} r_{i}
$$

with $r_{i}=\boldsymbol{P}^{-1} y_{i} \neq 0$. After a little manipulation, it can be found

$$
\left(\xi_{i} I-A_{c_{D}}\right)_{i}=P^{-1} \boldsymbol{D P}
$$

After some complex calculations we can find

$$
\frac{\left\|\left(\zeta_{i} \boldsymbol{I}-\boldsymbol{A}_{\boldsymbol{c}_{\boldsymbol{D}}}\right) \boldsymbol{r}_{i}\right\|_{2}}{\left\|r_{i}\right\|_{2}} \leq v\left(\boldsymbol{A}_{\boldsymbol{c}}\right)\|\boldsymbol{D}\|_{2}
$$

Since

$$
\|\boldsymbol{D}\|_{2}=\left\{\rho\left(\boldsymbol{D} \boldsymbol{D}^{*}\right)\right\}=\kappa
$$

then

$$
\frac{\left\|\left(\zeta_{i} \boldsymbol{I}-\boldsymbol{A}_{\boldsymbol{c}_{\boldsymbol{D}}}\right) r_{i}\right\|_{2}}{\left\|r_{i}\right\|_{2}} \leq v\left(\boldsymbol{A}_{\boldsymbol{c}}\right) \kappa
$$

Assume $\lambda_{c i}$ is characteristic value of matrix $\boldsymbol{A}_{\boldsymbol{c}}$ which has shortest distance to $\zeta_{i}$, then 


\section{Construction of controller candidate}

The parameter values used for the robotic manipulator are $m_{1}=1 \mathrm{~kg}, l_{1}=1 \mathrm{~m}$, $m_{\mathrm{e}}=2 \mathrm{~kg}, \quad \delta_{\mathrm{e}}=30^{\circ}, \quad I_{1}=0.12 \mathrm{~kg} \mathrm{~m}^{2}, \quad l_{\mathrm{c} 1}=0.5 \mathrm{~m}, \quad I_{\mathrm{e}}=0.25 \mathrm{~kg} \mathrm{~m}^{2}$, $l_{\text {ce }}=0.6 \mathrm{~m}$,

We propose the controller candidate as

$$
\boldsymbol{u}_{a}(\boldsymbol{x})=\boldsymbol{L} \boldsymbol{x}
$$

where $\boldsymbol{L} \in \mathfrak{R}^{2 \times 4}$.

The error between exact controller and approximation controller can be expressed as

$$
\boldsymbol{e}(\boldsymbol{x})=-\boldsymbol{\alpha}(\boldsymbol{x})-\boldsymbol{\beta}(\boldsymbol{x}) \boldsymbol{K} \boldsymbol{z}(\boldsymbol{x})+\boldsymbol{L} \boldsymbol{x}
$$

where

$$
\begin{aligned}
& \boldsymbol{\alpha}(\boldsymbol{x})=\boldsymbol{C}(\boldsymbol{q}, \dot{\boldsymbol{q}}) \dot{\boldsymbol{q}} \\
& \boldsymbol{\beta}(\boldsymbol{x})=\boldsymbol{H}(\boldsymbol{q})
\end{aligned}
$$

Based on (42) it found

$$
\varepsilon(x)=\beta^{-1}(x) e(x)
$$

After some complex calculations we can find

$$
\boldsymbol{\varepsilon}(\boldsymbol{x})=\left[\begin{array}{l}
\varepsilon_{1}(\boldsymbol{x}) \\
\varepsilon_{2}(\boldsymbol{x})
\end{array}\right]
$$

where

$$
\begin{aligned}
\varepsilon_{1}(\boldsymbol{x})= & \frac{\beta_{12} \alpha_{2}-\beta_{22} \alpha_{1}}{|\boldsymbol{\beta}(\boldsymbol{x})|}-\boldsymbol{K}_{1} \boldsymbol{x} \\
& +\left[\beta_{22}-\beta_{12}\right] \frac{\boldsymbol{L} \boldsymbol{x}}{|\boldsymbol{\beta}(\boldsymbol{x})|}
\end{aligned}
$$

and

$$
\begin{aligned}
\varepsilon_{2}(\boldsymbol{x})= & \frac{\beta_{21} \alpha_{1}-\beta_{11} \alpha_{2}}{|\boldsymbol{\beta}(\boldsymbol{x})|}-\boldsymbol{K}_{2} \boldsymbol{x} \\
& +\left[\begin{array}{ll}
-\beta_{21} & \beta_{11}
\end{array}\right] \frac{\boldsymbol{L} \boldsymbol{x}}{|\boldsymbol{\beta}(\boldsymbol{x})|}
\end{aligned}
$$

with

$$
\begin{aligned}
& \boldsymbol{K}_{1}=\left[\begin{array}{llll}
K_{11} & K_{12} & K_{13} & K_{14}
\end{array}\right] \\
& \boldsymbol{K}_{2}=\left[\begin{array}{llll}
K_{21} & K_{22} & K_{23} & K_{24}
\end{array}\right] \\
& \alpha_{1}(\boldsymbol{x})=\left(1.04 \sin x_{3}-0.60 \cos x_{3}\right) \\
& \left(-2 x_{2} x_{4}-x_{4}^{2}\right) \text {, } \\
& \alpha_{2}(\boldsymbol{x})=\left(1.04 \sin x_{3}-0.60 \cos x_{3}\right) x_{2}^{2} \text {, } \\
& \beta_{11}=3.34+2.08 \cos x_{3}+1.2 \sin x_{3} \text {, } \\
& \beta_{12}=0.97+1.04 \cos x_{3}+0.60 \sin x_{3} \text {, }
\end{aligned}
$$


$|\boldsymbol{\beta}(\boldsymbol{x})|=\beta_{11} \beta_{22}-\beta_{12} \beta_{21}$.

By differentiating $\boldsymbol{\varepsilon}(\boldsymbol{x})$ with respect to $\boldsymbol{x}$, and putting the value $\boldsymbol{x}=\mathbf{0}$, it can be found

$$
\begin{aligned}
& \frac{\partial \varepsilon_{1}}{\partial x_{1}}=90+\frac{0.97 L_{11}-2.01 L_{21}}{1.2173} \\
& \frac{\partial \varepsilon_{1}}{\partial x_{2}}=19+\frac{0.97 L_{12}-2.01 L_{22}}{1.2173} \\
& \frac{\partial \varepsilon_{1}}{\partial x_{3}}=\frac{0.97 L_{13}-2.01 L_{23}}{1.2173} \\
& \frac{\partial \varepsilon_{1}}{\partial x_{4}}=\frac{0.97 L_{14}-2.01 L_{24}}{1.2173} \\
& \frac{\partial \varepsilon_{2}}{\partial x_{1}}=\frac{-2.01 L_{11}+5.42 L_{21}}{1.2173} \\
& \frac{\partial \varepsilon_{2}}{\partial x_{2}}=\frac{-2.01 L_{12}+5.42 L_{22}}{1.2173} \\
& \frac{\partial \varepsilon_{2}}{\partial x_{3}}=132+\frac{-2.01 L_{13}+5.42 L_{23}}{1.2173} \\
& \frac{\partial \varepsilon_{2}}{\partial x_{4}}=32+\frac{-2.01 L_{14}+5.42 L_{24}}{1.2173}
\end{aligned}
$$

If we selected

$$
\boldsymbol{L}^{T}=\left[\begin{array}{ll}
-400.723 & -148.608 \\
-84.5971 & -31.3727 \\
-217.958 & -105.184 \\
-37.9775 & -18.3274
\end{array}\right]
$$

will imply

$$
\left.\frac{\partial \boldsymbol{\varepsilon}}{\partial \boldsymbol{x}}\right|_{\boldsymbol{x}=\mathbf{0}}=\mathbf{0}
$$

and furthermore this will imply

$$
\kappa=\left\|\left(\left.\left(\frac{\partial \boldsymbol{\varepsilon}(\boldsymbol{x})}{\partial \boldsymbol{x}}\right)^{T}(\nabla \boldsymbol{T}(\boldsymbol{x}))^{-1}\right|_{\boldsymbol{x}=\mathbf{0}}\right)^{T}\right\|=0 .
$$

Since $\kappa=0$, then for this case, Equation (16) is always true, so it can be concluded that the origin of closed loop system (1) under the controller

$$
\boldsymbol{u}_{a}(\boldsymbol{x})=\left[\begin{array}{ll}
-400.723 & -148.608 \\
-84.5971 & -31.3727 \\
-217.958 & -105.184 \\
-37.9775 & -18.3274
\end{array}\right]^{T} \boldsymbol{x}
$$

is asymptotically stable. 


\section{$J$ Implementation the proposed controller}

Let us define $\boldsymbol{e}=\boldsymbol{q}-\boldsymbol{q}_{\mathbf{d}}=\left[\begin{array}{ll}e_{1} & e_{2}\end{array}\right]^{T}$ as a tracking error vector. Implementation of approximating state feedback in robotic manipulator is arranged as follows

$$
\boldsymbol{\tau}=\left[\begin{array}{ll}
-400.723 & -148.608 \\
-84.5971 & -31.3727 \\
-217.958 & -105.184 \\
-37.9775 & -18.3274
\end{array}\right]^{T}\left[\begin{array}{c}
e_{1} \\
\dot{\theta}_{1} \\
e_{2} \\
\dot{\theta}_{2}
\end{array}\right]
$$

where $\boldsymbol{q}_{\mathbf{d}}$ is a desired output vector.

\section{Simulation Result and Discussion}

The best way to compare performance between the controller which is synthesized by exact feedback linearization and the controller which is synthesized by approximating state feedback linearization, is to use digital simulation. The simulation results are shown in Figure 2 - Figure 5.

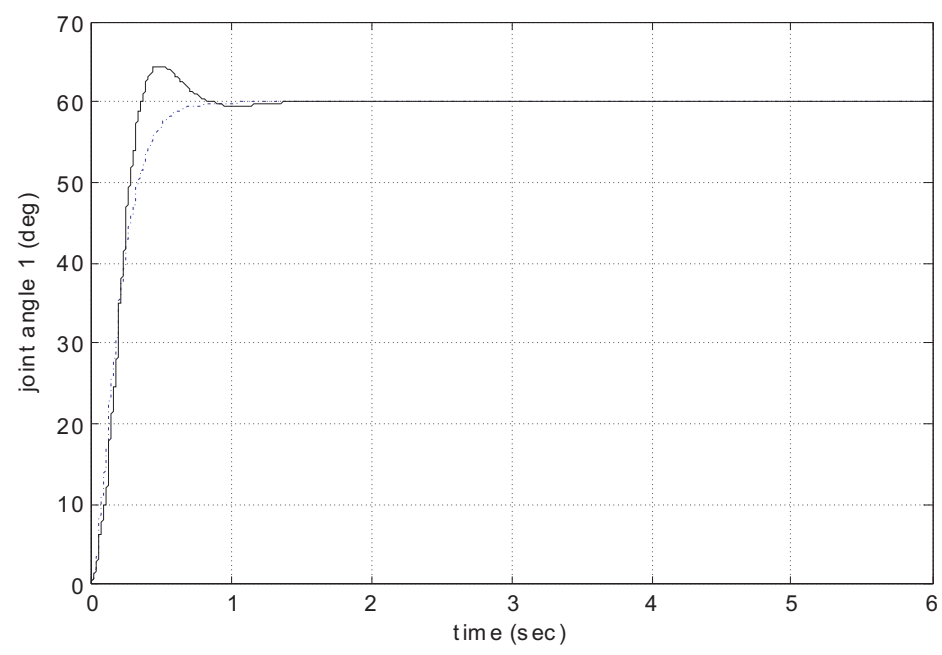

Figure 2. Response of joint angle 1.

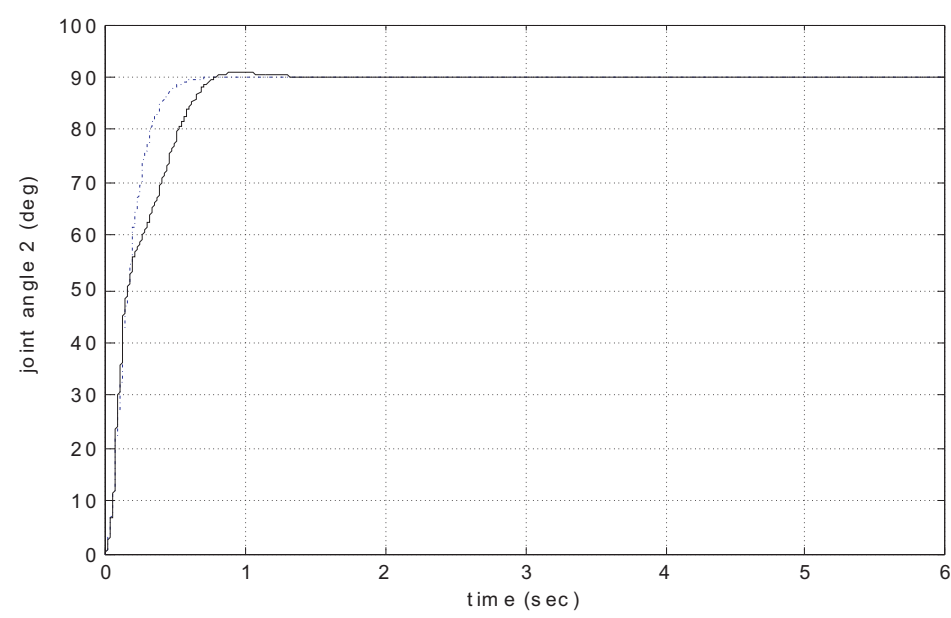

Figure 3. Response of joint angle 2 . 


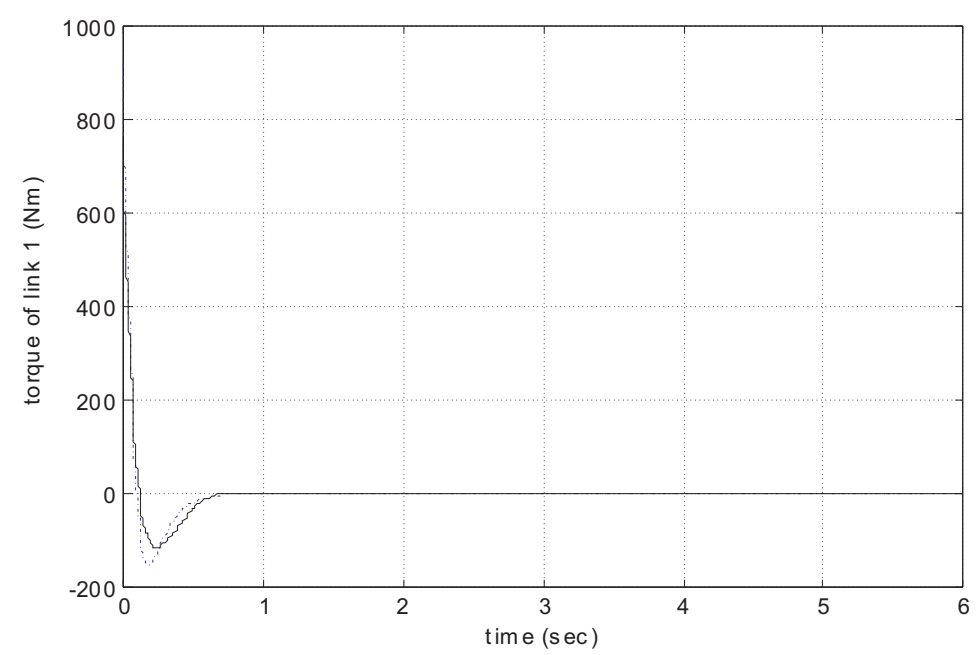

Figure 4. Control torque of joint 1.

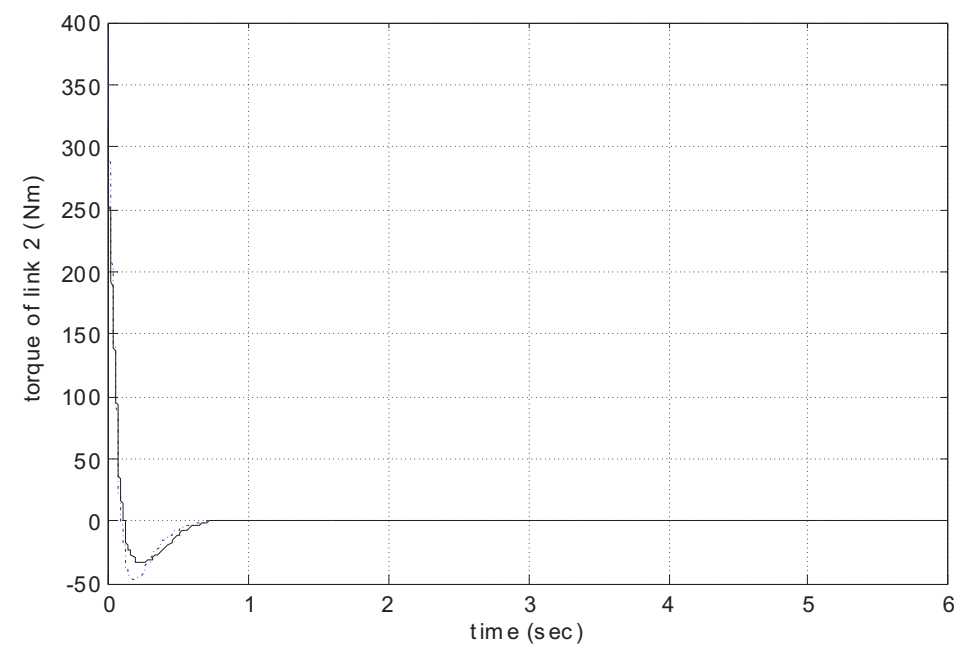

Figure 5. Control torque of joint 2.

The transient response of joint angle 1 under exact feedback linearization (solid) has no overshoot. This is similar to overdamped response of a second order linear system. The transient response of joint angle 1 under approximating feedback linearization (dashed dot) has $7.8 \%$ overshoot. This response is similar to underdamped response of a second order linear system. The control torque of joint 1 under exact feedback linearization (solid) has overshoot which is slightly larger than control torque of joint 1 under approximating feedback linearization (dashed dot).

The transient response of joint angle 2 under exact feedback linearization (solid) has no overshoot. This is similar to response of joint angle 1 . The transient response of joint angle 2 under approximating feedback linearization (dashed dot) has $0.95 \%$ overshoot. This response has slightly degraded performance. The control torque of joint 2 under exact feedback linearization (solid) has overshoot larger than control torque of joint 2 under approximating feedback linearization (dashed dot). The overshoot of torque response is used to push the joint angle 2 to achieve transient response without overshoot. 
Simulation results show that there is no significant performance degradation when using the proposed method.

Table 1 shows the comparison of the proposed method and the exact feedback linearization method.

Table 1. The comparison of the proposed method and the exact feedback linearization method.

\begin{tabular}{|c|c|c|}
\hline No & Proposed Method & $\begin{array}{c}\text { Exact Feedback } \\
\text { Linearization } \\
\text { Method }\end{array}$ \\
\hline 1 & Lin ear State Feedback & Nonlinear State Feedback \\
\hline 2 & Simple Function & Complexity Function \\
\hline 3 & $\begin{array}{l}\text { Practical Realization Is Easier } \\
\text { And Possible }\end{array}$ & $\begin{array}{l}\text { Practical Realization Is More } \\
\text { Difficult And May Be Impossible }\end{array}$ \\
\hline 4 & $\begin{array}{l}\text { Theoretical Response May Be } \\
\text { Slightly Degraded }\end{array}$ & Theoretical Response Is Optimal \\
\hline 5 & Practical Response Is Similar & Practical Response Is Similar \\
\hline
\end{tabular}

\section{Conclusions}

Exact feedback linearization is commonly used as a nonlinear controller. The main weak point of the exact linearization controller is that its implementation is difficult. This study presents a synthesis of approximating state feedback for robotic manipulator control system based on exact feedback linearization. The synthesis method is formulated by using Lyapunov theory, analysis of shifting characteristic values, and differential geometry.

The proposed method has four stages. First, the controller is synthesized by using exact feedback linearization. Second, the controller is replaced by the controller candidate which is synthesized by approximating an exact feedback controller. Third, stability of the controller candidate is verified by using Lyapunov theory. Fourth, the controller candidate is implemented by using digital simulation.

In case of a robotic manipulator controller, there is no significant performance degradation when approximating exact feedback linearization by the proposed method. The controller candidate has satisfied performance which is shown by a digital simulation.

\section{References}

[1] Bedrossian, N. S. and Spong, M. W. (1995), Feedback Linearization of Robot Manipulator and Riemannian Curvature, Journal of Robotic Systems, Vol. 12, No. 8,

[2] pp. 541-552.

[3] Boothby, W. A. (1975), An Introduction to Differentiable Manifolds and Riemanian Geometry, Academic Press, New York.

[4] Chen, C. T. (1970), Linear System Theory and Design, Holt-Saunders International Editions, New York.

[5] Chong, C., Yan. W., and Guangxiong, W. (1991), Nonlinear Decoupling Control and Exact Linearization of Induction Motor Drive Using Microprocessor, Proc. Of IECON '91, pp. 707-709.

[6] Fattah, H. A. (2000), Input-output Linearization of Induction Motors with Magnetic Saturation, Proc. Amer. Contr. Conf., pp:600-604.

[7] Goldberg, J. L. (1992), Marix Theory with Applications, McGraw-Hill, Singapore.

[8] Gray, P. R. and Meyer, R. G. (1977), Analysis and Design of Analog Integrated Circuits, John Wiley and Sons, New York.

[9] Isidori, A. (1995), Nonlinear Control Systems, 3rd Ed., Springer, New York.

[10] Khalil, H. K. (1992), Nonlinear Systems, Macmillan Publishing Company, New York. 
[1] Kim, C. S., Seo, W. H., Han, S. H., and Khatib, O. (2001) Fuzzy Logic Control of a Robot Manipulator Based on Visual Servoing, Proceedings of IEEE International Symposium on Industrial Electronics, ISIE 2001, Vol. 3, pp. 1597-1602.

[2] Lancaster, P. and Tismenetsky, M. (1985), The Theory of Matrices, second edition, Academic Press, London.

[3] La Salle, J. and Lefschetz, S. (1961), Stability by Lyapunov Direct Method, Academic Press, New York.

[4] Lotfazar, A., Eghtesad, M., and Mohseni, M. (2003) Integrator Backstepping Control of a 5 DOF Robot Manipulator Incorporating Actuator Dynamics, Proceedings of 2003 IEEE Conference on Control Applications, CCA 2003, Vol. 2, pp. 1007-1012.

[5] Mahayana, D. (1991), Sistem Pengaturan dengan Mikroprosesor, PAU Mikroelektronika.

[6] Mahayana, D. (1998), Sintesa Sistem Kontrol Nonlinier, Disertasi Doktor, Bandung Institute of Technology.

[7] Mokhtari, A., Benallegue, A., and Daachi, B. (2006), Robust Feedback Linearization and $H_{\infty}$ Controller for a Quad Rotor Unmanned Aerial Vehicle, J. Elect. Eng., Vol. 57, No. 1, pp. 20-27.

[8] Nurbambang, I. S. and Mahayana, D. (1990), Penguat Instrumentasi, PAU Mikroelektronika.

[9] Ogawa, M., Yamamoto, H., and Itoh, T. (1991), Tracking Controller Design for a Nonlinear CSTR Using Linearization, Proc. IECON, pp:2229-2234.

[10] Patino, H. D., Carelli, R., and Kuchen, B. R. (2002), Neural Networks for Advanced Control of Robot Manipulator, IEEE Transactions On Neural Networks, Vol. 13, No. 2, March, pp. 343-354.

[11] Purwar, S. (2007) Higher Order Sliding Mode Controller for Robotic Manipulator, IEEE 22nd International Symposium on Intelligent Control, ISIC 2007, pp. 556-561.

[12] Rangan, C. S., Sarma, G. R., and Mani, V. S. V. (1992), Instrumentation Devices and Systems, New Delhi, Tata McGraw Hill.

[13] Sadati, N., Ghadami, R., and Bagherpour, M. (2005), A Robust Adaptive Neural Network Controller Based on Variable Structure System, 5th International Conference on Technology and Automation, Thessalonixi, Greece, Oct.

[14] Shiller, Z. and Dubowsky, S. (1985) On The Optimal Control of Robotic Manipulator with Actuator and End-Effector Constraints, Proc. IEEE International Conference on Robotics and Automation, pp. 614-620.

[15] Slotine, J. E. and W. Li (1991), Applied Nonlinear Control, Pentice-Hall.

[16] Spong, W.S. and Groeneveld, T. (1997), An Experimental Evaluation of Riemannian Curvature Based Feedback Linearization for a Direct Drive Manipulator, IFAC-IFIPIMACS Conference on Control of Industrial Systems, Belfort, France, May, pp. 643-647.

[17] Torres, S. , Mendez, J. A., Acosta, L., and Becerra, V. M. (2007), On Improving The Performance in Robust Controllers for Robot Manipulator with Parametric Disturbances, Control Engineering Practice, Vol. 15, pp. 557-566.

[18] Yazarel, H. and Cheah, C.C. (2002), Task-Space Adaptive Control of Robotic Manipulator with Uncertainties in Gravity Regressor Matrix and Kinematics, IEEE Transactions on Automatic Control, Vol. 47, No. 9, pp. 1580-1585.

[19] Yong, K. D. (1978), Controller Design for a Manipulator Using Theory of Variable Structure System," IEEE Trans. Systs., Man, Cybern., Vol. SMC-8, No. 2, Feb, pp. 210 218.

[20] Yurkovich, S. (1992), Flexibility Effects on Performance and Control, Robot Control, Ed. M. W. Spong, F. L. Lewis, and C. T. Abdallah, IEEE Press, New York. 
Dimitri Mahayana, lecturer at STEI-ITB Doctor in Engineering Sciences (DR), in Electrical Engineering, Bandung Institute of Technology, Bandung, Indonesia 1998, Master of Engineering (M. Eng) from The Department of Electrical Engineering, Waseda University, Tokyo, Japan 1994, Bachelor of Engineering (Ir.) from the Department of Electrical Engineering, Institute of Technology Bandung 1989. He has papers Published in International and National Publication. 